\title{
BMJ Open Alternatives to continuous positive airway pressure treatment in sleep apnoeas and hypopnoeas syndrome related to myofunctional and postural reeducation therapy: protocol for a systematic review
}

\author{
Marta Maria Guijarro-Herráiz, ${ }^{1}$ Natalia María Arias-Palencia (D) ,2 \\ Lidia Lucas- de la Cruz, ${ }^{1}$ Beatriz Guijarro-Herráiz, ${ }^{3}$ Blanca Notario-Pacheco, ${ }^{1}$ \\ Rocio Palomo-Carrión (1D , ${ }^{4}$ Jose Luis García-Klepzig, ${ }^{5}$ Montserrat Solera-Martínez ${ }^{1}$
}

To cite: Guijarro-Herráiz MM, Arias-Palencia NM, Lucas- de la Cruz L, et al. Alternatives to continuous positive airway pressure treatment in sleep apnoeas and hypopnoeas syndrome related to myofunctional and postural reeducation therapy: protocol for a systematic review. BMJ Open 2021;11:e049286. doi:10.1136/ bmjopen-2021-049286

- Prepublication history for this paper is available online. To view these files, please visit the journal online (http://dx.doi. org/10.1136/bmjopen-2021049286).

Received 14 February 2021 Accepted 12 October 2021

Check for updates

(c) Author(s) (or their employer(s)) 2021. Re-use permitted under CC BY-NC. No commercial re-use. See rights and permissions. Published by BMJ.

For numbered affiliations see end of article.

Correspondence to Mrs Natalia María Arias-

Palencia; natalia.arias@uclm.es

\section{ABSTRACT}

Introduction The main treatment for sleep apnoeas and hypopnoeas syndrome (SAHS) is continuous positive airway pressure (CPAP). However, patients sometimes do not adhere to the treatment protocol. Supplementary and complementary therapies have appeared as alternatives. Some of the therapies which are especially important are those related to myofunctional (MFT) and postural therapy (PT), as all of them are non-invasive, and their application is simpler than that of CPAP. We aim to present a protocol for a systematic review and meta-analysis for investigating new SAHS treatments, including the protocols and frequency of use and the effects they have on patient signs, symptoms and quality of life.

Methods and analysis The literature search will be conducted using the Cochrane, Web of Science, Medline (via PubMed) and Scopus databases, from January 2020 to December 2020. All types of studies written in English and Spanish that investigate the use of alternative SAHS treatments related to MFT, or more importantly, the combination of MFT and PRT, will be selected. To evaluate their quality, the Critical Appraisal Checklist for Analytical Cross-Sectional will be applied. The primary factor valued in the studies will be the inclusion of MFT and PT reeducation in the treatment. Subgroup analyses will be carried out evaluating the specific type of treatments chosen and the improvements or deteriorations in the level of health and quality of life in the patients. Finally, several patient-related outcomes, namely sleep quality, quality of life and sleep Apnoeas and Hypoapnoeas Index, will be examined.

Ethics and dissemination In this case, ethical approval is not necessary. The data used in the review will be exclusively obtained from published studies, implying there are no privacy concerns. The information obtained will be relevant to understand if the new treatments applied in SAHS are effective, and if postural and MFT therapy used together can be considered an appropriate approach to treat this disease.

The results will be published in a peer-reviewed journal.
Strengths and limitations of this study

- This review will present an explicit methodology to perform a search for literature investigating alternative sleep apnoeas and hypopnoeas syndrome (SAHS) treatments.

- It includes postural reeducation therapy with myofunctional therapy for the treatment of SAHS.

- The publication of this protocol for a systematic review prevents unnecessary duplication of research.

- When the validity of a selected article is not clear, two independent researchers will decide. A third researcher will be consulted if consensus is not reached.

- A potential limitation of this research could be the lack of enough studies that meet all the established inclusion criteria.

\section{INTRODUCTION}

Sleep apnoeas and hypopnoeas syndrome (SAHS) is a public health problem and one of the most prevalent respiratory disorders. ${ }^{1}$ It is characterised by episodes of total (apnoea) or partial (hypoapnoea) collapse of the upper airway that can limit the passage of air to the lungs during sleep. ${ }^{2}$ This can have a significant impact on sufferers, for example, reducing quality of life, increasing daily tiredness, causing lack of concentration and resulting in cognitive decline. ${ }^{3}$

SAHS has also been recognised as a risk factor for cardiovascular disease, high blood pressure, coronary artery disease, cardiac arrhythmia, cerebrovascular accident and metabolic diseases (such as diabetes). ${ }^{3-5}$ The continuous positive airway pressure (CPAP) is the most important treatment for SAHS, 
especially for patients with moderate-to-severe SAHS, and it can completely abolish respiratory events. ${ }^{67}$ The inictial acceptance is fairly good and adherence is suboptimal. ${ }^{7}$ Another study found that about two third of the OSA patients of the study did not use the CPAP machine throughout the 4 years of the study. ${ }^{8}$ The main limitations of CPAP are its acceptance problems and a lack of continuous patient adherence in many cases. Therefore, other treatment alternatives are sought. ${ }^{9}$ In recent years, one of the most important advances has been the development of a multidisciplinary approach. It is known that invasive and non-invasive treatments exist. Regarding invasive therapies, examples include surgeries at the level of the palate, uvula or tongue. These can be defined as interventions that make some changes to anatomical structures. Along the same line, we find orthodontics, where mandibular advancement devices and other similar mechanisms are used. Regarding non-invasive treatments, the most relevant are myofunctional (MFT) and postural therapy (PT). These can be defined as interventions that, will affect function if they induce any change. Treatment with MFT significantly improves determining values of SAHS; for example, it decreases the sleep Apnoea and Hypoapnoea Index (AHI) in the CPAP registry and it improves snoring and sleep quality and the quality of life.$^{10}$ Intervention with MFT involves training exercices for the tongue, palate and facial and labial musculature. This has shown to be effective in reducing both the condition in the long-term and comorbidity indicators. In all cases, the intervention is limited to the facial and oral level. ${ }^{11}$

However, it is known that PRT is relevant in the treatment of SAHS patients. Many studies are focused on patient posture at night. These studies talk about the use of devices that are in contact with different parts of the patient's body and limit changes in position.

In recent publications, it has been observed that SAHS could also be associated with cancer incidence. Therefore, this syndrome presents a significant socioeconomic burden, wich would improve if it was possible to establish more specific evaluation methods, and more effective, precise and multidisciplinary treatments.

The aim is to ensure that patients spend as little time as posible in a supine position, since this is the worst position during sleep time.

The aim of this study was to provide a clear methodology to find evidence to support the use of all-day PT, not just during sleep, with SAHS patients. The evidence shows that intervention through the stomatognathic system and PT are valid alternatives as SAHS's treatments. ${ }^{12}$

\section{OBJECTIVES}

The aim of this protocol study is to present an objective and clear methodology to increase the knowledge and understanding of the use of MFT and PT in SAHS patiens. In particular, the effects of these alternative therapies on health indicators, comorbidity risks and patient quality of life will be investigated.

\section{METHODS AND ANALYSIS}

This systematic review and meta-analysis protocol has been registered in the International Prospective Register of Systematic Reviews database. The methodology of this protocol will be reported according to the Preferred Reporting Items for Systematic Reviews and Meta-analyses Protocols (PRISMA) and the Cochrane Collaboration Handbook for Systematic Reviews of Interventions will be used to report and guide the review methods. ${ }^{13}$ The literature search will be conducted using the Cochrane, Web of Science, Medline (via PubMed) and Scopus databases, from January 2020 to December 2020. A systematic review of the study is currently underway, focusing on treatment with MFT and PT in SAHS. The review is expected to be completed within a maximum period of 1 month. Once the review is completed, we will begin field work, and expect to have all the data analysed and the study completed within a year.

\section{Inclusion and exclusion criteria for study selection \\ Type of studies and interventions}

Studies will be selected from the literature by searching for those that discuss characteristics of the main alternative SAHS interventions and include interventions related to MFT or PT. The most important studies for this review will be those where both interventions are applied simultaneously. No differentiation will be made between type of studies, provided that the information is related to these types of interventions, although the most interesting types of studies for the present work will be systematic reviews.

\section{Type of participants in studies}

The protocol is focused on the adult population (aged 18-70), so we will consider excluding studies that are developed exclusively for populations under 18. The SAHS pathology is more prevalent in men than women; however, we want to include both genders, so we will consider excluding studies that are developed exclusively in one of the genders alone. It is important that participants are within 2 years of SAHS diagnosis and do not have any other pathologies, such as craniofacial or rachidian deformities, tumours, serious cognitive or intellectual decline, or drug use which may have possible secondary effects on sleep.

\section{Type of outcomes}

We will select the studies that include results related to quality of life, quality of sleep and AHI analysis in PSG. The following scales are the preferred ones to obtain the data from: The Functional Outcomes Sleep Questionnarie (FOSQ) or Sleep Apnoea Quality of life Index (SAQLI) and Quality of sleep through Epworth Sleepiness Scale (ESS).

Search strategy, the literature search will be conducted from January to December 2020 using several databases: The Cochrane Library, Web of Science, Medline (via Pubmed) and Scopus. Searches for unpublished studies 
Table 1 Search strategy for the MEDLINE database

\begin{tabular}{lll} 
Search terms & & \\
\hline sleep apnea hypopnea syndrome & AND & oral appliance therapy \\
OR apnoea & OR positional therapy & health related quality of life \\
OR OSA & OR sleep position & OR quality of life \\
OR sleep apnoea & OR treatment positional obstructive & OR quality life \\
OR sleep apnea syndrome & OR oropharyngeal exercises & OR sleep quality \\
OR obstructive apnea & OR positional treatment & \\
OR obstructive sleep apnoea & OR positional therapy & \\
OR central sleep apnoea & OR oral appliance treatment & \\
OR central sleep apnea & OR oral appliance & \\
OR positional central sleep apnea & OR sleep stages and position & \\
OR obstructive sleep apnea & OR oropharynx exercises & \\
hypopnea syndrome & OR myofunctional therapy & \\
\end{tabular}

will be conducted on OPEN GRAY, ProQuest dissertations and Thesis Global, Theseo, Networked Digital Library of Theses and Dissertations and Google Scholar. The search strategy using boolean criteria are defined in table 1.

Using previous reviews, we will explore the reference lists of included articles and retrieve studies that potentially meet the inclusion criteria. There will be no limitations on the date of publication or the location of the study. The literature search will be independently conducted by two reviewers, and disagreements will be solved by consensus or by involving a third researcher.

\section{Selection of studies and data extraction}

Two independent researchers will screen all relevant titles and abstracts and they will identify eligible studies for publication. Reviewers will analyse the full texts and choose all potentially eligible articles, based on exclusion and inclusion criteria.

The process of identifying, screening and including or excluding studies will be shown using the PRISMA $^{13}$ flow chart (figure 1).

The main characteristics of the studies will be extracted by the reviewers as follows: (1) name of the first author; (2) year of publication; (3) type of study; (4) country where the study was performed; (5) characteristics of the study population (age, sex, previous existing pathologies and number of participants in each group); (6) type of interventions used in the study population and (7) outcome measures (table 2).

To avoid the double counting of patients that have been included in more than one report by the same author or working group, the recruitment periods will be evaluated. When necessary, corresponding authors of the potentially included studies will be contacted to obtain any missing information.

\section{Assessment of risk of bias}

We will use the Quality Assessment Tool for Observational Cohort and Cross-Sectional Studies from the United States National Institute of Health National Heart, Lung and Blood Institute. ${ }^{14}$ This tool evaluates the risk of bias according to the following domains: quality of the research question, reporting of the population definition, participation rate, recruitment, sample size, appropriateness of statistical analyses, timeframe for associations, exposure levels, ascertainment of the exposure, appropriateness of the outcome measured, outcome blinding of

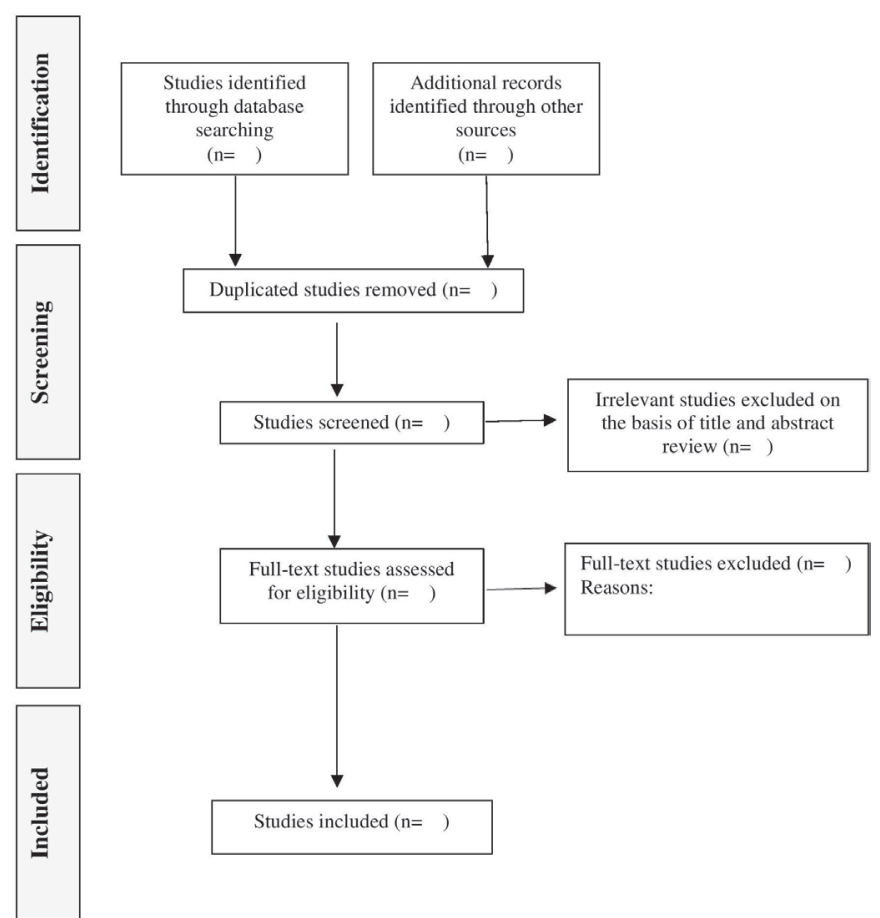

Figure 1 PRISMA flow diagram of identification, screening, eligibility and inclusion studies. PRISMA, Preferred Reporting Items for Systematic Reviews and Meta-analyses. 
Table 2 Collected characteristics of studies included in the systematic review

\begin{tabular}{llll}
\hline Study characteristics & $\begin{array}{l}\text { Population characteristics } \\
\text { and baseline }\end{array}$ & Intervention & Outcome \\
\hline First author's name & Age & Myofunctional therapy & Quality of life related wit SAHS \\
Country & Gender & Postural therapy & Sleep Apnoeas and Hypoapnoeas Index \\
Publication year & Sample size & & Sleep quality \\
Type & Previous pathologies & & \\
\hline
\end{tabular}

SAHS, sleep apnoeas and hypopnoeas syndrome.

researchers, lost to follow-up and confounding variables. The general bias of each study was considered as follows: 'good' if most criteria were met and with a low risk of bias; 'fair' if some criteria were met and with a moderate risk of bias; or 'poor' if few criteria were met and with a high risk of bias. Any disagreements over the assessment of quality will be solved by consensus. A third reseacher will be consulted if consensus is not reached.

\section{Statistical analysis}

After data extraction, the reviewers will decide whether meta-analysis is possible. At least four studies addressing MFT therapy and postural reeducation therapy as interventions for SAHS patients will be required to conduct the meta-analysis. If this is posible, all analyses will be performed using Comprehensive Metaanalysis Software (second version, Biostat, Englewood, NJ, USA) and StataSE software, V.15 (StataCorp). The standardised mean difference will be calculated for each study reporting the association between MFT therapy and postural reeducation therapy in SAHS intervention using Cohen's index ${ }^{15}$ to compute the pooled effect size estimates with $95 \%$ CIs fixed effects models. ${ }^{16}$

We will also provide further information on the main confounders of our research. Some confounders required to get full points of the quality assessment of the published studies are previous pathologies or kind of interventions that the patient has received. We can know the outcomes of our gold standard PSG and there are elements that can interfere with night rest.

The heterogeneity results across studies will be evaluated using the $\mathrm{I}^{2}$ statistic, and the following values will be used for its interpretation: $0 \%$ to $30 \%$ 'not important' heterogeneity; $>30 \%$ to $50 \%$ 'moderate' heterogeneity; $>50 \%$ to $80 \%$ 'substantial' heterogeneity, and $>80 \%$ to $100 \%$ 'considerable' heterogeneity. The corresponding $\mathrm{p}$ values will be also considered. A linear meta-analysis regression model will be used to explore whether covariates could be associated with the magnitude of the effects and explain the observed statistical heterogeneity. ${ }^{17}$

If a meta-analysis is not feasible, we will perform a narrative synthesis. $^{18}$

\section{Sensitivity analysis}

To assess the robustness of the findings, we will perform a sensivity analysis by removing studies one by one from the main analysis.

\section{DISCUSSION}

The aim of this protocol study is to present an objective and clear methodology to conduct a systematic review and meta-analysis investigating new, non-invasive SAHS treatments. The aim is to increase knowledge and understanding of the association between MFT and postural reeducation therapy related to SAHS treatments and their benefits on health indicactors, comorbidity risks and quality of life of patients.

The main treatment in the case of SAHS is CPAP. Studies about the adherence of patients to the CPAP treatment show that a variable percentage of them refer a fairly but not very good initial acceptance and suboptimal adherence to CPAP, ${ }^{19}$ so better results will probably be obtained by including new treatments such as those related to surgical techniques, orthodontics treatments, PT, or manual therapy for SAHS's treatment. ${ }^{2021}$

Treatment techniques focus on the stomatognathic system, aiming to recover normal muscular function by reeducating movement patterns and readapating respiratory ones. Work on these exercises, mainly at the cranial level (oral, pharyngeal and lingual), makes the symptoms of these patients and the parameters of PSG improve. For example, a decrease in AHI may be seen. Today, it is essential to implement PT in MFT. The human body can no longer be thought of as a set of isolated structures, but as a whole. This means that posture will influence the rest of the anatomical structures (spinal, facial and cranial level) and should be part of the MFT treatment. Numerous studies evaluate the results of the application of PT during sleep, using devices and systems that prevent patients from lying in a supine position. ${ }^{22} 23$ However, postural intervention outside the sleep hours is not considered.

We think that combining the application of both therapies would maximise the positive results in terms of disease management. We want to conduct the literature review to search for possible interventions where both therapies are documented.

Potential limitations of this research could include publication bias, information bias, inclusion of articles in English or Spanish only, poor statistical analysis and inadequate reporting of methods and findings in the primary studies.

To summarise, we will carry out a systematic review and possible meta-analysis, with the objective of reviewing 
existing literature on the simultaneous application of MFT therapy and PT in SAHS treatment. Additionally, the effect of these therapies on health indicators, comorbidity risks, PSG parameters and patient quality of life will be investigated. If the study confirms positive effects, it could encourage the application of this therapy to complement or supplement CPAP) treatment.

To be used in daily practice, new SAHS interventions need guidelines and recommendations based on rigorous and updated reviews summarising the available scientific evidence in order to improve the effectiveness of interventions, search for new therapeutic alternatives and improve quality of life. The findings of this systematic review could lead to an improvement in the health of patients with SAHS and will provide evidence of the benefit of a multidisciplinary approach to this pathology.

\section{ETHICS AND DISSEMINATION}

The data included in this project will be provided by original studies; therefore, ethical approval and informed consent of patients will not be required.

This protocol provides a clear and structured procedure to extract relevant and newflagled information about association of MFT and PT with SAHS's treatment. This study will have clinical health implications as it could provide new alternatives to patients diagnosed with SAHS: non-invasive interventions which might help to improve the results with CPAP's treatment or even be complementary to or substitute for this device.

The AHI through polysomnography will be used since this technic is considered to be the gold standard in the diagnosis of SAHS, and variations in its results are a clear indicator of improvement or worsening of SAHS. ${ }^{17-23}$ The FOSQ will be used since it is a generic instrument to measure health-related quality of life with sleep apnoea. This is an increasingly relevant factor as a way of studying the health of the population and of analysing the efficacy and effectiveness of health interventions. This questionnarie was designed as a simple option that could be administered under wide variety of measurement conditions (by mail, post, self-adminisetered or by interview), and it is valid for healthy subjects and with different health problems. ${ }^{24}{ }^{25}$ Another option is SAQLI, ${ }^{26}$ it has similar characteristics than the anterior scale and both are valid. Also, the ESS will be used because it is a simple, self-administered questionnaire which is shown to provide a measurement of the subject's general level of daytime sleepiness.

Suggestions for future research will be made according to the findings of this systematic review and meta-analysis (if it is possible). Evidence-based recommendations will be given to improve the quality of life and quality of sleep in these patients. Finally, longitudinal studies will be needed to confirm if the new therapeutic interventions on SAHS are a better option than its traditional treatment.

\section{Author affiliations}

${ }^{1}$ Faculty of Nursing, Universidad de Castilla-La Mancha, Cuenca, Spain

${ }^{2}$ Faculty of Education, Universidad de Castilla-La Mancha, Cuenca, Spain

${ }^{3}$ Internity Medicine, Hospital Virgen de la Luz, Cuenca, Spain

${ }^{4}$ Faculty of Phsysiotheray, Universidad de Castilla-La Mancha - Campus de Toledo, Toledo, Spain

${ }^{5}$ Facultad de Farmacia, Universidad Complutense de Madrid, Madrid, Spain

Twitter Natalia María Arias-Palencia @nati_jm

Contributors All authors comply with the recommendations of ICMJE, according to: MMG, NMAP and BN-P conceived and designed the protocol. LL, BG, RPC, JLG and MS participated in the development of the search strataegy. MS, LL, BG and MMG planned the data extraction. LL and MS tested the feasibility of the study and revised the manuscript. All authors have contributed to the final written manuscript.

Funding The authors have not declared a specific grant for this research from any funding agency in the public, commercial or not-for-profit sectors.

Competing interests None declared.

Patient consent for publication Not applicable.

Provenance and peer review Not commissioned; externally peer reviewed.

Open access This is an open access article distributed in accordance with the Creative Commons Attribution Non Commercial (CC BY-NC 4.0) license, which permits others to distribute, remix, adapt, build upon this work non-commercially, and license their derivative works on different terms, provided the original work is properly cited, appropriate credit is given, any changes made indicated, and the use is non-commercial. See: http://creativecommons.org/licenses/by-nc/4.0/.

\section{ORCID iDs}

Natalia María Arias-Palencia http://orcid.org/0000-0001-5004-3197

Rocio Palomo-Carrión http://orcid.org/0000-0003-4034-2585

\section{REFERENCES}

1 Santín M J, Jorquera A J, Jordán J, et al. [Long-term continuous positive airway pressure (CPAP) use in obstructive sleep apnea]. Rev Med Chil 2007;135:855-61.

2 Zonato Al, Bittencourt LR, Martinho FL. Association of Systematic Head and Neck Physical Examination With Severity of Obstructive Sleep Apnea???Hypopnea Syndrome. Laryngoscope 2003;113:973-80.

3 Álvarez-Sala Walter J, García Mochón L, González Núñez J. Evaluación sanitaria y socioeconómica del síndrome de apneas e hipopneas del sueño (SAHS) en España [Internet], 2020. Madrid: Alberto Alcocer. Available: http://ses.org.es/docs/libro-blancoapneas-hipopneas.pdf

4 Azagra-Calero E, Espinar-Escalona E, Barrera-Mora JM. Obstructive sleep apnea syndrome (OSAS). review of the literature. Med Oral Patol Oral Cir Bucal 2012;17:925-9.

5 Torre Bouscoulet L, López Escárcega E, Castorena Maldonado A, et al. [Continuous positive airway pressure used by adults with obstructive sleep apneas after prescription in a public referral hospital in Mexico City]. Arch Bronconeumol 2007;43:16-21.

6 Oksenberg A, Silverberg D, Offenbach D, et al. Positional therapy for obstructive sleep apnea patients: a 6-month follow-up study. Laryngoscope 2006;116:1995-2000.

7 Sarrell EM, Chomsky O, Shechter D. [Treatment compliance with continuous positive airway pressure device among adults with obstructive sleep apnea (OSA): how many adhere to treatment?]. Harefuah 2013;152:140-4.

8 Mediano O, Romero-Peralta S, Resano P, et al. Obstructive sleep apnea: emerging treatments targeting the genioglossus muscle. $J$ Clin Med 2019;8. doi:10.3390/jcm8101754. [Epub ahead of print: 22 10 2019].

9 Camacho M, Certal V, Abdullatif J, et al. Myofunctional therapy to treat obstructive sleep apnea: a systematic review and metaanalysis. Sleep 2015;38:669-75.

$10 \mathrm{Kim}$ SJ, Choi J, Park YH, et al. Positional therapy for the reduction of obstructive sleep apnea. Sleep Biol Rhythms 2011;9:150-6.

11 Rodríguez-Romero B, Jiménez JM, Ares GP. Síndromes Y reeducación posturales en Los Trastornos temporo mandibulares. Revista Iberoamericana de Fisioterapia y Kinesiología 2004;7:83-98.

12 Moher D, Shamseer L, Clarke M, et al. Preferred reporting items for systematic review and meta-analysis protocols (PRISMA-P) 2015 statement. Syst Rev 2015;4:1. 
13 National Heart, Lung, and Blood Institute. Study quality assessment tools, 2019. Available: https://www.nhlbi.nih.gov/health-topics/studyquality-assessment-tools

14 Morris SB. Estimating effect sizes from pretest-posttest-control group designs. Organ Res Methods 2008;11:364-86.

15 Leonard T, Duffy JC. A Bayesian fixed effects analysis of the Mantel-Haenszel model applied to meta-analysis. Stat Med 2002;21:2295-312.

16 Higgins J. Cochrane Handbook for systematic reviews of interventions version. The Cochrane Collaboration, 2011: 2-3.

17 Higgins JPT, Thompson SG. Quantifying heterogeneity in a metaanalysis. Stat Med 2002;21:1539-58.

18 Abad Fernández A, de Miguel Díez J. [Therapeutic alternatives to CPAP in the sleep apnea-hypopnea syndrome. Scientific evidence]. Rev Clin Esp 2009;209:433-8.

19 Eijsvogel MM, Ubbink R, Dekker J, et al. Sleep position trainer versus tennis ball technique in positional obstructive sleep apnea syndrome. J Clin Sleep Med 2015;11:139-47.

20 Zonato Al, Bittencourt LR, Martinho FL, et al. Association of systematic head and neck physical examination with severity of obstructive sleep apnea-hypopnea syndrome. Laryngoscope 2003;113:973-80.
21 Clebak KT, Demetriou TJ, Carey S. Positional therapy for obstructive sleep apnea. Am Fam Physician 2020;101:16-17.

22 van Maanen JP, Richard W, Van Kesteren ER, et al. Evaluation of a new simple treatment for positional sleep apnoea patients. J Sleep Res 2012;21:322-9.

23 Rodríguez-Pérez V, Valencia-Flores M, Reyes-Lagunes I, et al. Adaptación y validación psicométrica del Cuestionario de Consecuencias Funcionales del Dormir (Functional Outcomes Sleep Questionnaire [FOSQ]) en habitantes de la Ciudad de México. Salud Ment 2013;36:307-13.

24 Vidal S, Ferrer M, Masuet C. Valores de la versión española del Cuestionario del Impacto Funcional del Sueño (FOSQ) en sujetos sanos $\mathrm{Y}$ en pacientes Con apnea obstructiva del sueño. Archivos de Bronconeumología 2007;43:256-61.

25 Catalán P, Martínez A, Herrejón A, et al. Internal consistency and validity of the Spanish version of the quality of life questionnaire specific for obstructive sleep apnea: sleep apnea quality of life index. Arch Bronconeumol 2012;48:431-42.

26 Ibáñez V, Silva J, Cauli O. A survey on sleep questionnaires and diaries. Sleep Med 2018;42:90-6. 